\title{
Extracorporeal Membrane Oxygenation in Refractory Cardiogenic Shock
}

\author{
Weimin Li, Dongyan Yang \\ Department of Cardiovascular Surgery, Tianjin Chest Hospital affiliated to Tianjin University, Tianjin, China
}

\section{ABSTRACT}

Background: Many clinicians do not know under what exact conditions extracorporeal membrane oxygenation $(\mathrm{ECMO})$ can get the best results. In this study, we explored the optimal indications for ECMO in patients with refractory cardiogenic shock.

Methods: From October 2014 to November 2019, 23 patients with refractory cardiogenic shock were treated with ECMO in our hospital, including 11 cases with acute left anterior myocardial infarction, 3 with acute left inferior and right ventricular myocardial infarction, and 9 with fulminant myocarditis. These cases were divided into survivors $(\mathrm{n}=$ $10)$ and nonsurvivors $(n=13)$, and the clinical data of the 2 groups were compared.

Results: The weaning rate of ECMO was $60.9 \%$. The discharge survival rate was $43.5 \%$. There were significant differences in age, sequential organ failure assessment (SOFA) score, vasoactive-inotropic (VIS) score, lactic acid concentrations, primary disease, and smoking history between survivors and nonsurvivors before ECMO $(\mathrm{P}<.05)$. There were significant differences in blood pressure (systolic and diastolic), oxygen partial pressure, and left ventricular ejection fraction between survivors and nonsurvivors 1 day before the removal of ECMO $(\mathrm{P}<.05)$.

Conclusions: The reversibility of the primary disease causing refractory cardiogenic shock is critical to the survival rate of ECMO. Etiological treatment is essential, and extra attention should be paid to the use of ECMO in patients with irreversible primary disease. ECMO should be regarded as a first aid device and is not suitable for long-term cardiac assistance; left ventricular assist or heart transplantation is a better option.

\section{INTRODUCTION}

The complex and unpredictable effects of extracorporeal membrane oxygenation (ECMO) on heart function, as well as its low survival rate in refractory cardiogenic shock, are confusing to clinicians. Quite a few conflate ECMO with traditional cardiopulmonary bypass, arguing that ECMO can

Received August 14, 2020; received in revised form October 10, 2020; accepted October 12, 2020.

Correspondence: Weimin Li, Department of Cardiovascular Surgery, Tianjin Chest Hospital affiliated to Tianjin University, 261, Taierzhuangnan Road, Finnan District, Tianjin, Cbina 300222; 8613920964244 (e-mail: lifengshu@163.com). partially or completely replace the function of heart and lungs and allow the organs to rest. However, this is not always the case. Traditional cardiopulmonary bypass is connected in parallel to the body's circulatory system, so blood does not pass through the heart and lungs, reducing the load on and resting them. ECMO is connected in series to the body's circulatory system, and the effects on the heart and lungs are therefore complex and subject to many factors. It is difficult to predict whether the heart and lungs get rest or benefit from ECMO. Studies of the effects of ECMO on the heart and lungs have often been contradictory and do not go into great detail about how to properly use ECMO. We report the cases of ECMO in our center and explore the optimal indications for ECMO in patients with refractory cardiogenic shock.

\section{METHODS}

From October 2014 to November 2019, 23 patients (14 males and 9 females, median age 55.00 years; interquartile range $48.00,68.00)$ with refractory cardiogenic shock were treated with ECMO in our hospital. Eleven patients had acute left anterior myocardial infarction, 3 acute left inferior and right ventricular myocardial infarction, and 9 fulminant myocarditis. Cases were divided into survivors $(\mathrm{n}=10)$ and nonsurvivors $(\mathrm{n}=13)$, and the characteristics of the 2 groups were compared.

In our center, venoarterial ECMO for refractory cardiogenic shock was indicated when the following criteria were met: persistence or aggravation of tissue hypoxia (extensive skin mottling, anuria, neurological impairment, elevated blood lactate, etc.) despite adequate fluid loading; or sustained hypotension (systolic blood pressure $<90 \mathrm{mmHg}$ or mean arterial pressure $<65 \mathrm{mmHg}$ ) despite infusion of very-highdose catecholamines (epinephrine $\geq 0.3 \mu \mathrm{g} / \mathrm{kg} / \mathrm{min}$, dopamine $\geq 15 \mu \mathrm{g} / \mathrm{kg} / \mathrm{min}$, norepinephrine $\geq 0.3 \mu \mathrm{g} / \mathrm{kg} / \mathrm{min}$ ).

Coronary angiography was carried out in 21 patients (the exceptions were 2 patients with fulminant myocarditis). Percutaneous coronary intervention (PCI) was carried out in 7 patients with acute left anterior myocardial infarction and 3 with acute left inferior and right ventricular myocardial infarction ( 9 before the establishment of ECMO, and 1 at the same time). Coronary artery bypass grafting (CABG) was carried out in 4 patients with acute left anterior myocardial infarction (3 before the establishment of ECMO, and 1 at the same time).

Venoarterial ECMO with femoral cannulation was used in all cases. ECMO support consisted of a polimethylpentene oxygenator and a centrifugal pump (Maquet, Hechingen, 
Germany). Activated clotting time of 180 to 210 seconds was maintained throughout the ECMO support period. The pump blood flow was adjusted to provide a cardiac index of $2.6 \mathrm{~L} / \mathrm{min} / \mathrm{m}^{2}$ or higher to obtain an inlet venous saturation $>70 \%$. Percutaneous vessel cannulation was carried out with a modified Seldinger technique in 5 cases; surgical vessel cannulation was carried out in the remaining cases.

The weaning process from venoarterial ECMO was performed by gradually decreasing ECMO support. ECMO was reduced to low levels (ECMO flow 1.0 to $1.5 \mathrm{~L} / \mathrm{min}$ ) with stable mean arterial pressure $\geq 65 \mathrm{mmHg}$, no or low doses of catecholamines (epinephrine $\leq 0.03 \mu \mathrm{g} / \mathrm{kg} / \mathrm{min}$, dopamine $\leq 5$ $\mu \mathrm{g} / \mathrm{kg} / \mathrm{min}$, norepinephrine $\leq 0.03 \mu \mathrm{g} / \mathrm{kg} / \mathrm{min}$ ), and central venous oxygen saturation $\geq 70 \%$.

Intra-aortic balloon pump (IABP) was applied in cases with acute myocardial infarction unless there was limited access to the femoral artery, such as PCI via the femoral artery on one side, ECMO or serious femoral arterial disease on the other (10 cases). For patients with fulminant myocarditis, IABP was used only if there was no pulsatile blood flow after ECMO (5 cases).

Categorical data are presented as percentages, and continuous data are presented as mean \pm standard deviation. Normally distributed continuous variables were compared using unpaired Student's $t$ test. Other continuous variables were compared using Mann-Whitney $U$ test. Categorical data were compared using $\chi^{2}$ test or Fisher's exact test. $P<$ .05 was considered statistically significant. All analyses were performed using SPSS PASW Statistics 22/Windows (IBM Corp., Armonk, NY).

All procedures performed in the study involving human participants were in accordance with the 1964 Helsinki Declaration and its later amendments or comparable ethical standards. This study was approved by the institutional review board, and written informed consent was waived because of the retrospective study design. No funding was acquired.

\section{RESULTS}

The interval between the onset of cardiogenic shock and venoarterial ECMO was 2.50 hours (range 2.50 to 3.50 ). The weaning rate of ECMO was $60.9 \%$. The discharge survival rate was $43.5 \%$ : all 11 patients with acute left anterior myocardial infarction died; all 3 patients with acute left inferior and right ventricular myocardial infarction survived; and 7 of 9 patients with fulminant myocarditis survived.

Of the 11 patients with acute left anterior myocardial infarction, 7 died on ECMO (1 brain death attributed to massive cerebral hemorrhage, 6 multiple system organ failure). Four patients were weaned off ECMO and cardiogenic shock recurred, and they died in the hospital. Two patients with complete atrioventricular block died, and the others recovered sinus rhythm at the later stages of ECMO.

Of the 7 patients with fulminant myocarditis accompanied by complete atrioventricular block, 5 were implanted with temporary pacemakers. Of those, 1 recovered autogenous pulsatile blood flow 5 days after beginning ECMO but suffered a massive cerebral hemorrhage and died on ECMO. Of the 2 cases with complete atrioventricular block who did not receive temporary pacemakers, 1 recovered sinus rhythm 5 days after beginning ECMO, and 1 had poor ECMO venous drainage, with circulation that could not be maintained effectively, and died on ECMO.

A comparison of baseline characteristics between survivors and nonsurvivors before ECMO is shown in Table 1. There were significant differences in age, SOFA score, VIS score, lactic acid concentrations, primary disease, and smoking history $(P<.05)$. The interval between the onset of cardiogenic shock and venoarterial ECMO showed no significant difference $(P>.05)$.

A comparison of characteristics between the groups while on ECMO is shown in Table 2. Lactic acid concentrations were significantly different 1 day after beginning ECMO $(P<.05)$. Systolic blood pressure and left ventricular ejection fraction were significantly different 2 days after beginning ECMO $(P<.05)$. Blood pressure (systolic and diastolic), oxygen partial pressure, and left ventricular ejection fraction were significantly different 1 day before the removal of $\operatorname{ECMO}(P<.05)$.

A comparison of other characteristics is shown in Table 3. The application of IABP showed a significant difference $(P<$ $.05)$. The application of continuous renal replacement therapy, the running times of ECMO and ventilator, the resident time in the intensive care unit, and complications showed no significant differences $(P>.05)$.

\section{DISCUSSION}

Cardiogenic shock is a low-cardiac-output state resulting in life-threatening end-organ hypoperfusion and hypoxia. Clinical presentation is typically characterized by persistent hypotension unresponsive to volume replacement, accompanied by clinical features of end-organ hypoperfusion requiring intervention with pharmacological or mechanical support [Thiele 2012; Reynolds 2008]. Efforts to reduce mortality from cardiogenic shock have been directed toward improving mechanical circulatory support devices [Thiele 2012; Sandhu 2015; Thiele 2015]. Venoarterial ECMO with femoral cannulation is first-line therapy, because it is easy to set up, and quick insertion at the bedside is possible in emergent settings. Rates of ECMO used for cardiogenic shock have gradually increased over the past decade, but the overall survival rate is still low [Nasr 2019].

There is a lot of debate about whether the technology of ECMO itself is flawed, along with indications, patient selection, timing, or method used. ECMO is not quite in line with the body's normal physiological condition: there is competitive blood flow, the afterload of the heart is increased, the preload of the heart is reduced, and whether the total load of the heart is reduced is difficult to assess. In addition, owing to competitive blood flow, it is difficult to determine whether coronary arteries are supplied by relatively hyperoxic blood via ECMO or by relatively hypoxic blood via the patient's own pulmonary circulation. Considering the huge suction 
Table 1. Patient characteristics before ECMO, by survival*

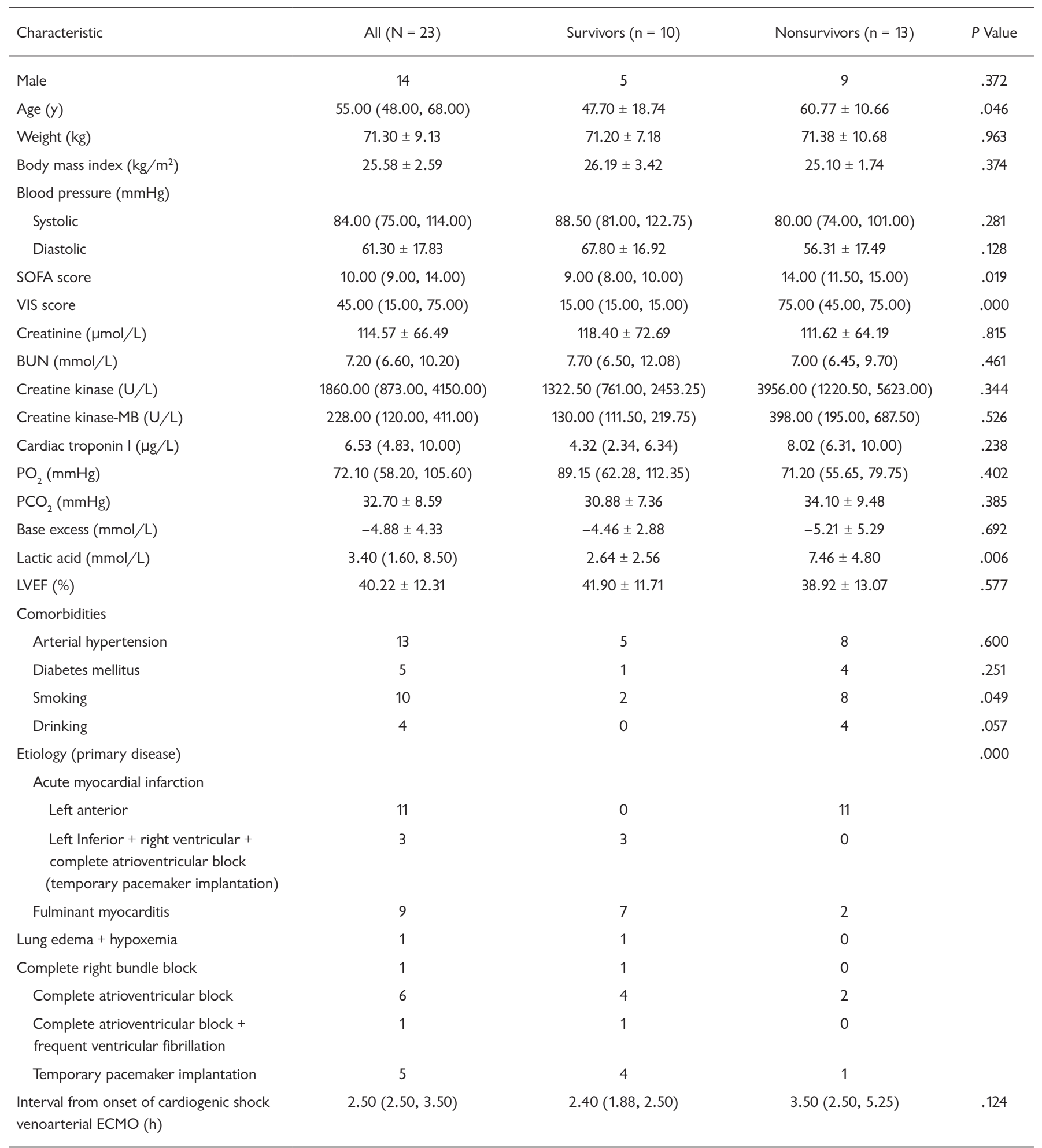

*Data are $\mathrm{n}(\%)$, mean \pm standard deviation, median (interquartile range), or $\mathrm{n}$.

BUN indicates blood urea nitrogen; LVEF, left ventricular ejection fraction; $\mathrm{PCO}_{2}$, carbon dioxide partial pressure; $\mathrm{PO}_{2}$, oxygen partial pressure. 
Table 2. Patient characteristics during ECMO, by survival*

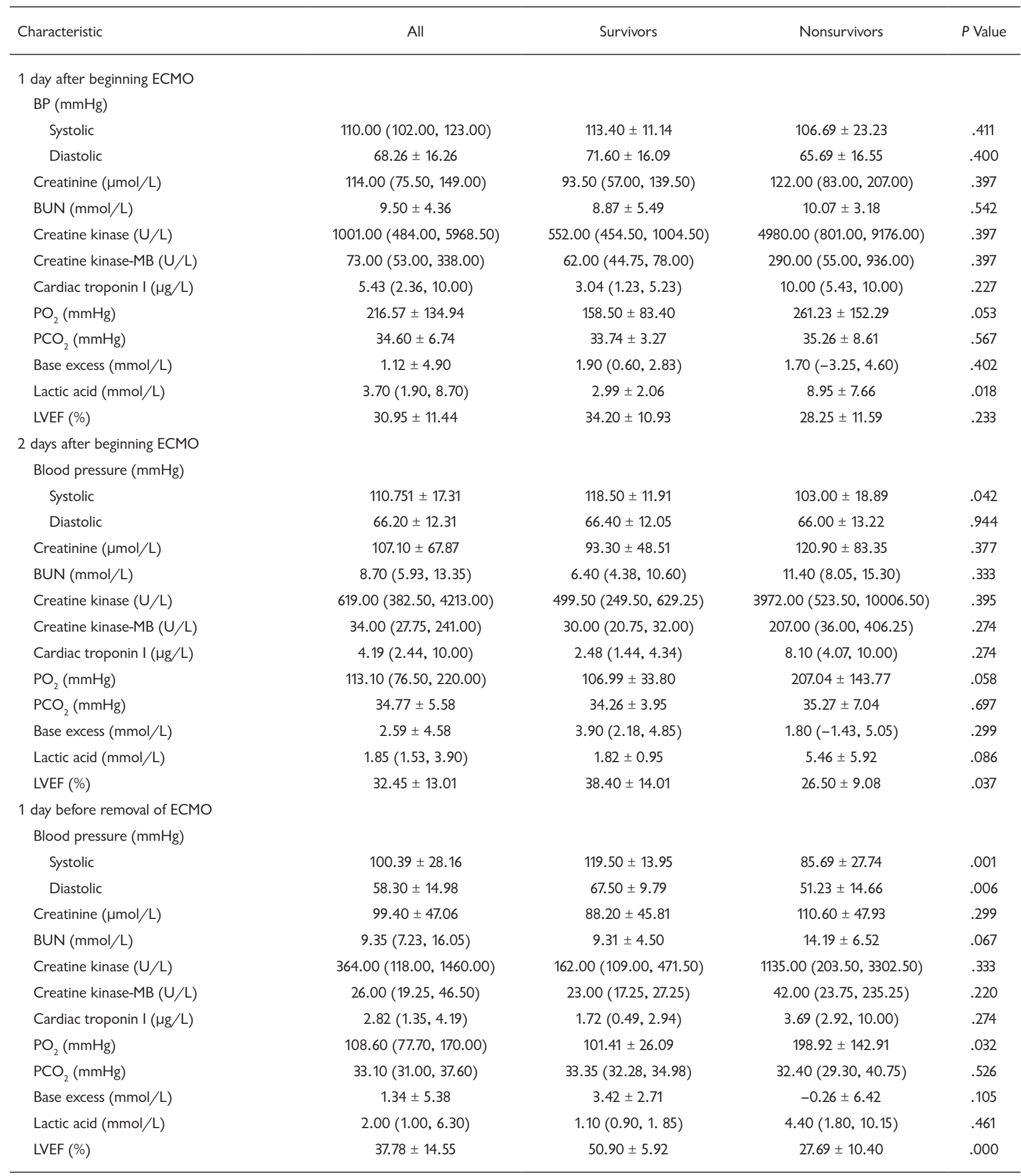

*Data are $\mathrm{n}(\%)$, mean \pm standard deviation, median (interquartile range), or $\mathrm{n}$.

$\mathrm{BUN}$ indicates blood urea nitrogen; LVEF, left ventricular ejection fraction; $\mathrm{PCO}_{2}$, carbon dioxide partial pressure; $\mathrm{PO}_{2}$, oxygen partial pressure 
Table 3. Other patient characteristics, by survival*

\begin{tabular}{|c|c|c|c|c|}
\hline Venoarterial ECMO weaning & 14 & 10 & 4 & .002 \\
\hline \multicolumn{5}{|l|}{ Cause of death } \\
\hline Multiorgan failure & 7 & 0 & 7 & \\
\hline Cardiogenic shock recurrence & 4 & 0 & 4 & \\
\hline Application of CRRT & 2 & 0 & 2 & .212 \\
\hline Application of IABP & 15 & 4 & 11 & .026 \\
\hline Running time of ECMO $(\mathrm{h})$ & $98.61 \pm 68.80$ & $92.10 \pm 28.42$ & $103.62 \pm 89.49$ & .669 \\
\hline Running time of ventilator $(h)$ & $110.00(73.00,135.00)$ & $116.00(93.75,133.50)$ & $80.00(37.00,259.00)$ & .461 \\
\hline Thrombosis of venous cannula & 1 & 0 & 1 & .393 \\
\hline Complication of implantation ischemia & 2 & 0 & 2 & .212 \\
\hline Brain bleed & 2 & 0 & 2 & .212 \\
\hline Upper gastrointestinal bleeding & 2 & 1 & 1 & .854 \\
\hline
\end{tabular}

*Data are $\mathrm{n}(\%)$, mean \pm standard deviation, or median (interquartile range).

CRRT indicates continuous renal replacement therapy.

effect of ECMO's venous drainage catheter, it is impossible to accurately assess whether the nutrients produced by gastrointestinal tract absorption and liver metabolism can reach the heart (and other organs) to provide necessary nutrients. Recently, particular attention was paid to the oxygen supply to the brain, which was assessed by several maneuvers [Rejmstad 2017]. Adjusting the pattern of ECMO connection, such as the veno-arterial-veno model, may ensure oxygen supply to the brain.

Our clinical data showed that blood pressure (systolic and diastolic), oxygen partial pressure, and left ventricular ejection fraction had significant differences between survivors and nonsurvivors 1 day before the removal of $\operatorname{ECMO}(P<.05)$. It was thus obvious that the reversibility of the primary disease causing refractory cardiogenic shock was critical to the survival rate in ECMO patients. We discuss separately the different assistant effects of ECMO in different primary diseases.

The causes of refractory cardiogenic shock are different, and acute myocardial infarction with left ventricular dysfunction is the most frequent cause of cardiogenic shock [Thiele 2012]. Advances in reperfusion therapy have been associated with improvements in survival, but in-hospital mortality remains high [Thiele 2012; Kolte 2014; Goldberg 2016]. The prognosis of acute myocardial infarction of different ventricular walls is different, and the relevant literature is sparse.

Hemodynamic changes of cardiogenic shock are not the same between left inferior plus right ventricular myocardial infarction and left anterior myocardial infarction. In general, the infarct-related artery of left inferior and right ventricular myocardial infarction is the right coronary artery, and that of left anterior myocardial infarction is the left coronary artery. The course and prognosis are also different [Lee 2014; Smarz 2016; Chen 2016].

In all 3 cases with acute left inferior and right ventricular myocardial infarction, ECMO was particularly suitable for this condition of cardiogenic shock. ECMO could effectively reduce the preload of the heart, which is usually significantly increased in left inferior and right ventricular myocardial infarction, and the increased afterload of the heart produced by ECMO could be compensated for by the relatively well-preserved left ventricle. During ECMO, physiological parameters gradually improved; in particular, urine output increased. A few days after successful PCI, complete atrioventricular block was restored to sinus rhythm, right ventricular myocardial infarction recovered, cardiogenic shock disappeared, weaning of ECMO was easy, and all 3 cases were discharged.

Left anterior myocardial infarction with cardiogenic shock was quite different. In all 11 cases with acute left anterior myocardial infarction, the left ventricular ejection fraction was generally lower and could not be improved despite PCI or CABG. The weak left ventricle received the increased afterload caused by ECMO, which further reduced cardiac output. The aortic valves could not open effectively, and as 
a result, left ventricular end-diastolic pressure significantly increased, left ventricular end-diastolic volume increased, left atrial pressure increased, pulmonary edema increased, gradual consolidation of the lung appeared, and further irreversible deterioration of vital organs ensued. Weaning of ECMO was very difficult. Therefore, ECMO is not suitable for longterm cardiac assistance in left anterior myocardial infarction. IABP, Impella pump, or atrial septotomy may be used for left ventricular decompression, but the results are often unsatisfactory. It is recommended that long-term left ventricular assist devices or heart transplantation be performed before irreversible damage occurs to other organs. In this condition, there is no value in trying to prolong ECMO, because the situation will only get worse, frequently resulting in multiple system organ failure. All 11 patients with acute left anterior myocardial infarction finally died in our hospital.

Fulminant myocarditis often presents similarly to acute coronary syndrome, and coronary angiography can be necessary to exclude acute ischemia as the cause of chest pain or cardiogenic shock. In patients with fulminant myocarditis with cardiogenic shock not responding to pharmacologic therapy, IABP can be used. If it is not effective in maintaining adequate cardiac output, then ECMO should be instituted. The use of the IABP in peripheral venoarterial ECMO is debated, however. In our study, IABP was used only if there was no pulsatile blood flow after ECMO. Most fulminant myocarditis is generally self-limited and just needs supportive treatment to recover; only a small percentage of patients need immunosuppressive therapy, develop chronic cardiac insufficiency, or require long-term cardiac assistance or heart transplantation [Montero 2018; Matsumoto 2018]. In fact, ECMO for fulminant myocarditis is not exactly consistent with the patient's physiological condition because competitive blood flow is still present, but most fulminant myocarditis is self-limited and brief and the survival rate is relatively high [Chong 2018; Liao 2018]. ECMO could improve the survival rate of patients with fulminant myocarditis and shorten the recovery time of sinus rhythm in patients with complete atrioventricular block or ventricular tachycardia [Lin 2016]. In general, ECMO had an excellent performance with this disease. In 9 cases with fulminant myocarditis, 7 were discharged.

In our opinion, the timely use of ECMO is indicated when life-threatening or irreversible damage to vital organs is imminent. However, during ECMO, it is necessary to make timely adjustments according to the progression of the disease and the different assistant effects of ECMO. For patients with reversible left inferior and right ventricular infarction or fulminant myocarditis, ECMO should be continued until recovery; for patients with irreversible left anterior myocardial infarction or fulminant myocarditis, there is no value in trying to prolong ECMO. It should be converted to left ventricular assist or heart transplantation in time.

This study was retrospective, highlighting a time when long-term left ventricular assist devices or heart transplantation was not available in most regions of China, so we had an opportunity to observe and discuss the effect of ECMO as the first, main, and ultimate cardiac assist device in refractory cardiogenic shock. Moreover, the different prognosis of acute myocardial infarction of different ventricular walls treated by ECMO allowed us to discuss the cases separately to guide future clinical treatment.

This study had several limitations. First, because of the small sample size, statistical analysis might lead to bias, and statistical analysis of different diseases could not be carried out. Second, because the patients were in emergency conditions, there was not much time to collect complete clinical data. Finally, because the study was retrospective, the criteria for diagnosis and treatment might not be uniform.

\section{CONCLUSION}

The reversibility of the primary disease causing refractory cardiogenic shock is critical to the survival rate in ECMO. Etiological treatment is essential, and extra attention should be paid to the use of ECMO in patients with irreversible primary disease. In that case, it should be regarded as a first aid device and is not exactly suitable for long-term cardiac assistance; left ventricular assist or heart transplantation is a better option.

\section{REFERENCES}

Chen ZW, Yu ZQ, Yang HB, et al. Rapid predictors for the occurrence of reduced left ventricular ejection fraction between LAD and non-LAD related ST-elevation myocardial infarction. BMC Cardiovasc Disord 2016;16:3

Chong SZ, Fang CY, Fang HY, et al. Associations with the in-hospital survival following extracorporeal membrane oxygenation in adult acute fulminant myocarditis. J Clin Med 2018;7:E452.

Goldberg RJ, Makam RC, Yarzebski J, McManus DD, Lessard D, Gore JM. Decade-long trends (2001-2011) in the incidence and hospital death rates associated with the in-hospital development of cardiogenic shock after acute myocardial infarction. Circ Cardiovasc Qual Outcomes 2016;9:117-125

Kolte D, Khera S, Aronow WS, et al. Trends in incidence, management, and outcomes of cardiogenic shock complicating ST elevation myocardial infarction in the United States. J Am Heart Assoc 2014;3:e000590.

Lee SN, Hwang YM, Kim GH, et al. Primary percutaneous coronary intervention ameliorates complete atrioventricular block complicating acute inferior myocardial infarction. Clin Interv Aging 2014;9:2027-2031.

Liao X, Li B, Cheng Z. Extracorporeal membrane oxygenation in adult patients with acute fulminant myocarditis: Clinical outcomes and risk factor analysis. Herz 2018;43:728-732.

Lin KM, Li MH, Hsieh KS, et al. Impact of extracorporeal membrane oxygenation on acute fulminant myocarditis-related hemodynamic compromise arrhythmiain children. Pediatr Neonatol 2016;57:480-487.

Matsumoto M, Asaumi Y, Nakamura Y, et al. Clinical determinants of successful weaning from extracorporeal membrane oxygenation in patients with fulminant myocarditis. ESC Heart Fail 2018;5:675-684.

Montero S, Aissaoui N, Tadié JM, et al. Fulminant giant-cell myocarditis on mechanical circulatory support: Management and outcomes of a French multicentre cohort. Int J Cardiol 2018;253:105-112. 
Nasr VG, Raman L, Barbaro RP, et al. Highlights from the Extracorporeal Life Support Organization Registry: 2006-2017. ASAIO J 2019;65:537-544.

Rejmstad P, Johansson JD, Haj-Hosseini N, Wardell K. A method for monitoring of oxygen saturation changes in brain tissue using diffuse reflectance spectroscopy. J Biophotonics 2017;10:446-455.

Reynolds HR, Hochman JS. Cardiogenic shock: Current concepts and improving outcomes. Circulation 2008;117:686-697.

Sandhu A, McCoy LA, Negi SI, et al. Use of mechanical circulatory support in patients undergoing percutaneous coronary intervention:
Insights from the National Cardiovascular Data Registry. Circulation 2015;132:1243-1251.

Smarz K, Zaborska B, Jaxa-Chamiec T, Tysarowski M, Budaj A. Right ventricular systolic function as a marker of prognosis after ST-elevation inferior myocardial infarction 5-year follow-up. Int J Cardiol 2016;221:549-553.

Thiele H, Ohman EM, Desch S, Eitel I, de Waha S. Management of cardiogenic shock. Eur Heart J 2015;36:1223-1230.

Thiele H, Zeymer U, Neumann FJ, et al. IABP SHOCK II Trial Investigators. Intraaortic balloon support for myocardial infarction with cardiogenic shock. N Engl J Med 2012;367:1287-1296. 